\title{
Eventos adversos en una nueva unidad de cuidados intensivos. Influencia del diseño y la tecnología de las instalaciones en las tasas de incidencia
}

Pablo Álvarez-Maldonado, ${ }^{1 *}$ Grisel Hernández-Ríos, ${ }^{1}$ Arturo Reding-BernaR y Raúl Cicero-Sabido ${ }^{1}$ ${ }^{1}$ Servicio de Neumología y Cirugía de Tórax; 'Dirección de Investigación. Secretaría de Salud, Hospital General de México "Dr. Eduardo Liceaaga", Ciudad de México, México

\section{Resumen}

Introducción: Nuevos hospitales están reemplazando a instalaciones antiguas. Existe poca información del desempeño de una unidad de cuidados intensivos (UCI) cuando es reubicada en un área nueva y equipada. Objetivo: Analizar el impacto del cambio de instalaciones de un ambiente compartido a camas individuales en la ocurrencia de eventos adversos en la Método: Estudio transversal, comparativo, con datos prospectivos de pacientes ingresados del 1 de marzo de 2014 al 28 de febrero de 2017 a la antigua UCI (aUCl) y del 17 de julio de 2017 al 17 de enero de 2019 a la nueva UCI (nUCI) de un hospital-escuela público. La tasa de eventos adversos se midió en eventos por 1000 días-paciente. Resultados: En $\overline{\$ 1} 188$ pacientes (aUCl, $n=681$ versus $n U C l, n=507$ ) se observó reducción en la tasa de paro cardiaco no previsto (razón de tasas 0.31, IC $95 \%=0.12-0.80$ ) e incremento en la tasa de extubación no planeada (razón de tasas 2.49, IC 95 \% = 1.24-5.01), estadísticamente significativos; los otros nueve eventos adversos monitoreados no mostraron cambios. Conclusiones: $\bar{C} o m-$ parada con la aUCl, la mayor parte de eventos adversos monitoreados no se modificaron significativamente en los 18 mêses de inicio de actividades de la nUCl.

PALABRAS CLAVE: Eventos adversos. Nuevas instalaciones. Unidad de cuidados intensivos.

\section{Abstract}

Introduction: New hospitals are replacing old facilities. There is little information on the performance of an intensive carejunit (ICU) when it is relocated in a new and equipped area. Objective: To analyze the impact of the change of ICU facilities from a shared environment to individual beds on the occurrence of adverse events. Method: Cross-sectional, comparative study, with prospectively collected data from patients admitted from March 01, 2014 to February 28, 2017 to the former ICU (f-ICU) and from July 17, 2017 to January 17, 2019 to the new ICU (n-ICU) of a public teaching hospital. The rate of adverse events was measured in events per 1,000 patient-days. Results: Among 1,188 patients ( $f-I C U, n=681$ vs. $n-I C U, n=507)$, a reduction in the rate of unforeseen cardiac arrest (rate ratio: $0.31 ; 95 \%$ confidence interval $[\mathrm{Cl}]=0.12-0.80$ ) and an increase i⿱亠凶禸 the rate of unplanned extubation (rate ratio: $2.49 ; 95 \% \mathrm{Cl}=1.24-5.01$ ) were observed, with both being statistically significant. 은 other nine monitored adverse events showed no changes. Conclusions: In comparison with the f-ICU, most of the monitored adverse events did not significantly change within the first 18 months of activities at the n-ICU.

KEY WORDS: Adverse events. New facilities. Intensive care unit.

Correspondencia:

*Pablo Álvarez-Maldonado

E-mail: pamyacs@yahoo.com
Fecha de recepción: 24-07-2019

Fecha de aceptación: 05-09-2019

DOI: 10.24875/GMM.19005421
Gac Med Mex. 2019;155:613=618 Disponible en PứbMed www.gacetamedicademexico.com 


\section{Introducción}

Los eventos adversos son responsables de 400000 muertes por año en pacientes hospitalizados en Estados Unidos; ocurren en el rango de 2 a 4 millones cuando no son letales. ${ }^{1}$ Las unidades de cuidados intensivos ( $\mathrm{UCl}$ ) reciben pacientes vulnerables que son tratados por múltiples proveedores, con tecnologías sofisticadas, en condiciones estresantes y en ambientes muchas veces inapropiados que promueven la aparición de errores, por lo que a menudo se les denomina "entornos hostiles". ${ }^{2,3}$ El diseño de las instalaciones puede desempeñar un papel clave en la mitigación de los aspectos hostiles de la $\mathrm{UCl}^{4-6} \mathrm{~A}$ medida que se construyen nuevos hospitales para reemplazar edificios antiguos se están construyendo $\mathrm{UCl}$ con tecnología renovada y habitaciones individuales en lugar de los tradicionales espacios abiertos para varios pacientes. ${ }^{7}$ Se ha descrito una asociación fuerte entre el diseño, los procesos de atención y los resultados cuando se cuenta con cualidades de estructura como habitaciones individuales, espacio y nueva tecnología en la $\mathrm{UCl}^{8}$

Dotada de instalaciones que datan de inicios de la centuria de 1900 y de equipamiento no acorde con los estándares actuales, la Unidad de Cuidados Intensivos Respiratorios (UCIR) del Hospital General de México "Dr. Eduardo Liceaga" (el primer hospital de especialidades de México) fue reubicada en julio de 2017 en un edificio completamente nuevo y equipado dentro del hospital. El objetivo principal de este estudio es investigar el impacto de la reubicación de la UCIR en la ocurrencia de EA que son monitoreados rutinariamente.

\section{Método}

Estudio transversal con datos obtenidos prospectivamente en una base de datos local, en el que se comparan las tasas de EA ocurridas en el periodo de 18 meses de inicio de actividades de la nueva UCIR (nUCIR), del 17 de julio de 2017 al 17 de enero de 2019, con las tasas de un periodo previo de tres años correspondiente a la UCIR antes de su reubicación (aUCIR), del 1 de marzo de 2014 al 1 de marzo de 2017.

Se incluyen datos de pacientes que ingresaron a la UCIR del Hospital General de México "Dr. Eduardo Liceaga", hospital-escuela público con 1131 camas. La UCIR, una de ocho UCI dentro del hospital, cuenta con personal de salud compuesto por un médico intensivista o neumólogo certificado en cada uno de sus turnos. La relación enfermera:paciente es de 1:1 a 1:3 y de enfermera intensivista:paciente de 1:3 a 1:6. Además cuenta con inhaloterapeutas tres repartidos en distintos turnos) y un promedio de cinco residentes rotatorios de las especialidades de neum logía, medicina interna, urgencias médicas, anestesiología o medicina crítica. El equipo multidisciplinario de la nUCIR es el mismo con el que contaba la UCIR en su antiguo edificio.

\section{Principales diferencias de diseño y equipamiento entre las $\mathrm{UCI}$}

La antigua UCIR (aUCIR) estuvo situada en un ărea adaptada para su uso en un edificio que data de principios de la centuria de 1900 , originalmente destinado para la atención de pacientes con tuberculosis. Contāba con un espacio común con siete camas no automáticas, separadas por cortinas de material sintético en un área total de $146 \mathrm{~m}^{2}$, una sola puerta de acceso para pacientes, familiares y personal de salud, que además seervía para ventilación (entrada de aire), un lavabo al ingreso de la unidad, una tarja al centro de la unidad, un baño con inodoro para el desecho de líquidos orgánieos, equipamiento consistente en monitores Spaceläbs ${ }^{\circledR}$ mCare-300 en cada cama con opción de visualización de presiones invasivas y ventiladores mecánicos Avea ${ }^{\circledR}$ (Viasys) para ventilación invasiva, adquiridos en 2009 durante la pandemia de influenza A H1N1. Contabacon dos sillones reclinables para uso común de los pacientes hospitalizados. No disponía de un área de descanso para médicos ni área administrativa.

La nUCIR se sitúa en el nuevo edificio para la atención de enfermedades cardiorrespiratorias y vasculares, fue inaugurada en julio de 2017 tras un periodo de construcción de tres años. La nUCIR cuenta con un área de $530 \mathrm{~m}^{2}$, con 12 cubículos independieñtes completamente aislados con puertas de vidrio corredizas y paredes, así como ventanas que dan al exterior. Las tomas de electricidad, aire medicinal, oxígeno y equipamiento están situadas en la pared posterīor a la cabecera del enfermo y cada cubículo cuenta con lavabo, además de una cama totalmente automática (InTouch $^{\circledR}$, Striker), un sillón reclinable, un monitoơ de signos vitales (IntelliVue MX800 ${ }^{\circledR}$, Philips) con opciones de monitorización de gasto cardiaco por método PiCCO, capnografía en tiempo, índice biespectral; variación de presión de pulso entre otros y ventilädor mecánico con capacidad para proporcionar ventilación invasiva y no invasiva (Puritan Bennett ${ }^{\mathrm{TM}}$ 840). Hay una entrada con lavabo a manera de transfer para 
Tabla 1. Definiciones de eventos adversos

Extubación accidental

Neumotórax

Paro cardiaco no previsto

Reintubación

Cambio del tubo endotraqueal

Caídas

Úlceras por presión

Hemorragia por úlcera por estrés

Trombosis venosa profunda-embolia pulmonar

Atelectasia

Muerte en $\mathrm{UCl}$
Retiro del tubo endotraqueal accidental por parte de un médico, una enfermera o cualquier otrō personal de atención médica en la $\mathrm{UCl}$ que no se ajuste a un programa o protocolo o extracción accidental del tubo endotraqueal inducida por el mismo paciente.

Presencia de aire o gas en la cavidad pleural que provoca colapso pulmonar de cualquier magnitud durante la estancia en $\mathrm{UCl}$ secundaria a punción vascular, toracentesis o barotraum Paro cardiaco inesperado que se produce en un paciente sin deterioro clínico en las últimas 24 horas de estancia en la UCI. Requiere la evaluación por parte del personal médico de turn un médico experimentado de la UCl.

Reintubación en las 48 a 72 horas posteriores a la extubación planificada.

Necesidad de reemplazar el tubo endotraqueal en el paciente previamente intubado debido oclusión o disfunción en cualquier sitio de la cánula.

Descenso no planificado al piso (o extensión del piso, por ejemplo, bote de basura u otro equipo con o sin lesiones. Se incluyen todos los tipos de caídas, ya sea por razones fisiológicas o ambientales.

Lesiones que pueden ser clasificadas en un grado o estadio o de profundidad desconocida localizadas en la piel o tejido subyacente como resultado de la presión, o la presión en combinación con la tensión en la piel que surge durante la estancia del paciente en la UCI.

Sangrado gastrointestinal significativo con inestabilidad hemodinámica o requerimiento de transfusión derivados de la enfermedad de la mucosa gastrointenstinal relacionada con el estres

Trombosis venosa profunda o embolismo pulmonar documentado clínica y radiológicamente origina durante la estancia del paciente en la $\mathrm{UCl}$.

Colapso lobar o pulmonar total debido a cualquier causa durante la estancia del paciente en la $\mathrm{UCl}$. No se incluyen las atelectasias segmentarias o "lineales".

Muerte secundaria a cualquier causa durante la estancia del paciente en la UCI. Los pacientes con orden de no reanimación y aquellos con otras medidas terapéuticas de limitación o retiro de্টু tratamiento no están incluidos. personal de salud y otra para familiares, así como una puerta de acceso exclusiva para pacientes en camilla. La nUCIR también dispone de un área de descanso para médicos y una oficina administrativa dentro de la misma unidad. Toda la unidad cuenta con aire acondicionado, inyector y extractor de aire. Además, para el envío y recepción de muestras biológicas y medicamentos hay un sistema neumático que comunica con las diferentes áreas dentro del edificio.

El procesamiento de gasometrías y la toma de radiografías portátiles con equipos disponibles in situ fueron características de la aUCIR y la nUCIR.

\section{Recolección de datos y análisis estadístico}

Los datos recabados prospectivamente fueron tomados de la base de datos DeDUCIR, ${ }^{9}$ una base de datos computarizada local en la que se registra información demográfica, de gravedad y pronóstico, además de EA y variables para el cálculo de indicadores de calidad, entre otra información. Los datos de la aUCIR fueron obtenidos de un estudio previo en un periodo en que fue implementado un programa multifacético de mejora de la calidad ${ }^{10} \mathrm{y}$ de la base de datos DeDUCIR. Las definiciones de EA se muestran en la Tabla 1. Las tasas de EA se calcularon en eventos por 1000 días-paciente. Las características sociodemográficas y clínicas de los pacientes se describen mediante valores absolutos y relativos (porcentajes) para variables dicotómicas y mediana y rango intercuartílico (percentiles 25 a 75) para las variables ๕ontinuas que no mostraron una distribución noťnal según la prueba de Kolmogorov-Smirnov. Las características de los pacientes se compararon con la prueba de chi cuadrada de Pearson para las variables dicotómicas y la prueba de la suma de rangos de Wilcoxon para las variables continuas, con la finalídad de analizar bivariadamente posibles diferencias estadísticamente significativas. Para comparar las tâsas de EA entre la nUCIR y la aUCIR se usó la razón de tasas con un intervalo de confianza (IC) de $95 \%$. 未as pruebas estadísticas se realizaron con el progrăma Stata ${ }^{\circledR}$, versión 15 (StataCorp LLC, Texas, Estad́dos Unidos). En este estudio no se reporta informaeión confidencial de los pacientes y su elaboración sēplaneó dentro de los reportes periódicos de calidad de la 
Tabla 2. Características sociodemográficas y clínicas de los pacientes en ambos periodos

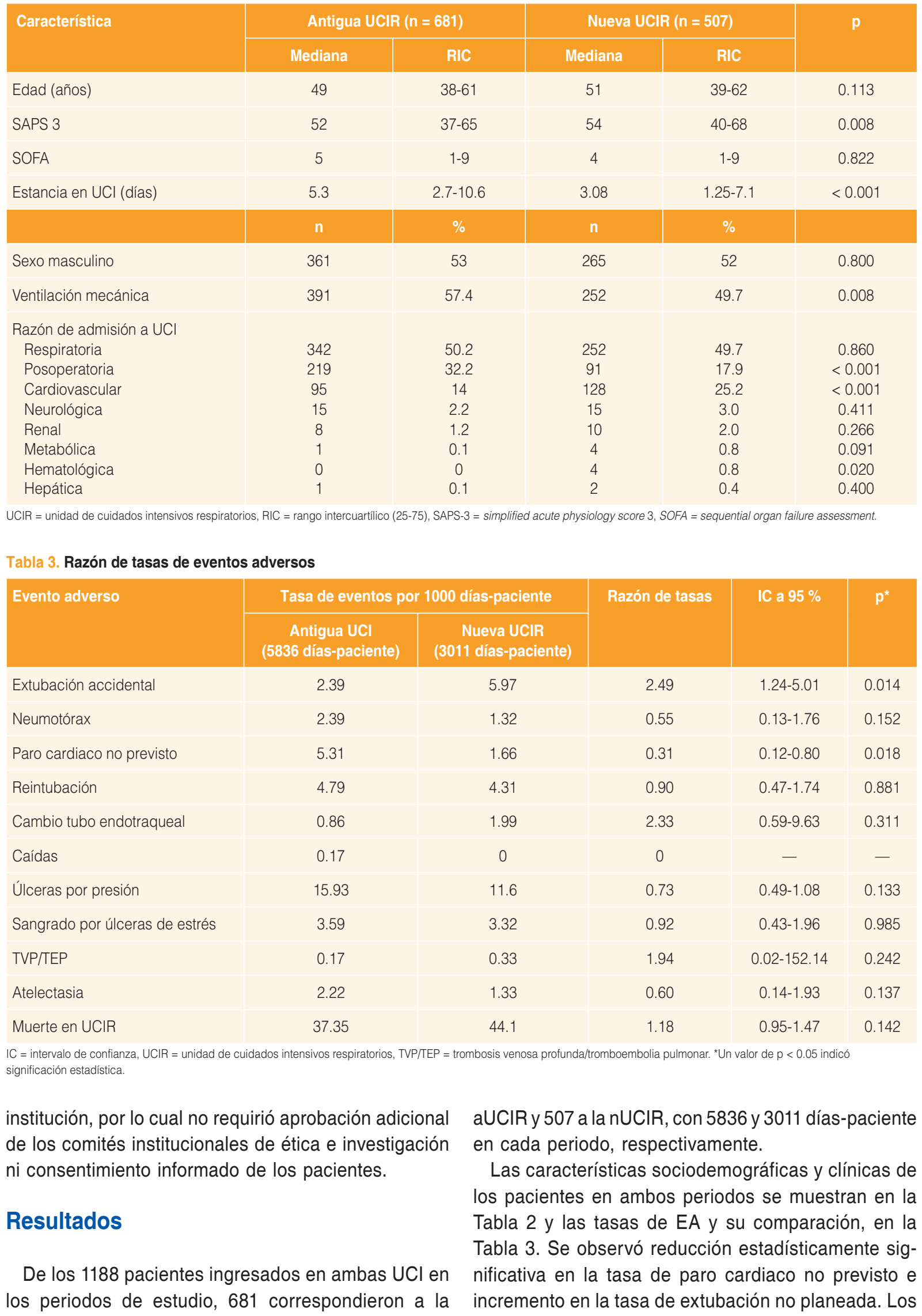


restantes nueve EA monitoreados no mostraron diferencias estadísticamente significativas. De acuerdo con el número de pacientes incluidos en cada periodo, se observó $49 \%$ más ingresos por mes durante el periodo correspondiente a la nUCIR, en comparación con el de atención en la aUCIR. La mortalidad en la $\mathrm{UCI}$ no fue diferente en ambos periodos cuando se hizo el análisis comparando tasas de eventos adversos, sin embargo, se identificó diferencia en la mortalidad cuando fueron comparadas las proporciones de fallecidos entre periodos en la aUCIR y la nUCIR: mortalidad de $32 \%$ versus $26.2 \%$, respectivamente $(p=0.036)$.

\section{Discusión}

Cualquier daño al paciente que resulte del manejo médico se considera un evento adverso. ${ }^{11}$ Los pacientes de la $\mathrm{UCI}$ son particularmente propensos a los EA dada su condición inestable y requerimiento de múltiples intervenciones. Atributos del diseño de la UCI como luz y espacio apropiados, control del ruido y habitaciones individuales han demostrado una fuerte asociación con mejores resultados en la supervivencia. ${ }^{8}$ También se ha observado que estos atributos junto con el uso de nuevas tecnologías pueden impactar favorablemente en los procesos de atención. ${ }^{12}$ Pese a buscar una asociación entre el cambio estructural y de equipamiento de la UCIR con menor ocurrencia de errores, en este análisis la mayoría de los EA monitoreados no se modificaron significativamente, con excepción de la tasa de paro cardiaco no previsto, que disminuyó, y la tasa de extubación no planeada, que se incrementó.

En todo el mundo, las antiguas instalaciones de salud están siendo reemplazadas o renovadas para adaptarse a los nuevos requerimientos de los servicios de atención médica, incluidos el aumento del número de camas, los avances tecnológicos y la necesidad de privacidad de los pacientes. ${ }^{7,13}$

Debido a las condiciones únicas de los pacientes de la UCI es razonable suponer que la reubicación y la posterior transición del personal a un nuevo entorno de trabajo puede ser más compleja que para otros departamentos del hospital. La transición es un proceso que requiere adaptación al entorno y se considera uno de los proyectos de gestión de cambios más complejos. ${ }^{14,15}$ Un estudio que evaluó la percepción del personal de salud cuando un hospital se mudó de habitaciones compartidas a habitaciones individuales encontró que el nuevo modelo incrementó la carga de trabajo para médicos y enfermeras y que el estrés en el personal fue en aumento durante los 15 messes posteriores al cambio. ${ }^{16}$ En un estudio más reciente, Lin et al. ${ }^{7}$ identificaron preocupaciones del persônal de salud por la seguridad del paciente al reubicấrse en una UCl geográfica y estructuralmente nuevaceon habitaciones individuales, entre las que resaltanqque las enfermeras fueran incapaces de obtener la ayuda necesaria, la pérdida de supervisión de personafơnenos experimentado y el requerimiento de mayor üso de sedación y restricciones físicas para evitar eventos como la autoextubación. En efecto, estos factores pudieron haber influido en la tasa de extubación no planeada en el análisis que se presenta, que tuvö un incremento significativo. Además de las limitacionnes del campo visual de vigilancia y distancia hacia los pacientes que impuso la nueva estructura de la UE়IR, el personal médico y de enfermería fue el mismo.para dar servicio a casi el doble de camas y $49 \%$ más ingresos por mes.

Es necesario considerar que en la aUCIR durante el periodo analizado fue implementado un progrăma multifacético de mejora de la calidad que derivờ en reducción en cinco de once tasas de EA y mayor supervivencia. ${ }^{10}$ Aunque los pacientes de la nÚC|R fueron pacientes más graves a su ingreso (segư la escala SAPS 3) y con más condiciones cardiovascưlares, tuvieron menores tiempos de ventilación mecánica y de estancia en la $\mathrm{UCl}$, sin que la mortalidad variase significativamente cuando se consideró como evento adverso (eventos por 1000 días-paciente). Más aún, cuando se comparan las proporciones de defunciones en ambos periodos (como habitualmente se hace para evaluar el rendimiento clínico de una UCI o un servicio hospitalario), la mortalidad fue menor en el periodo correspondiente al arranque de actividades dè la nUCIR. Por ello creemos que la UCIR tuvo una trănsición exitosa al nuevo edificio y que es posible reducir significativamente las tasas de EA y otros indicadores con la implementación de proyectos de mejora, el cambio organizacional y la contratación de personal sufficiente acordes con la nueva estructura. ${ }^{10,17-19}$

En relación con la disminución significativa de la tasa de paro cardiaco no previsto, atribuimos êste hecho a la posibilidad de que con más y mejjor tecnología en la nUCIR y sistemas de alarmas más sensibles, los cambios en la condición clínica gue preceden a estos eventos pudieron haber sido reconocidos tempranamente, a diferencia de los demás EA. Se sabe que el monitoreo continuo de siğnos vitales con equipamiento de vigilancia adecuado 
aminora la posibilidad de muerte inesperada secundaria a complicaciones prevenibles en pacientes hospitalizados. ${ }^{20,21}$

Existen algunas limitaciones de este estudio que merecen comentarse, como la dificultad para saber cuántos días los pacientes estuvieron en riesgo de padecer cada evento adverso. Además, el análisis corresponde a una sola $\mathrm{UCl}$ con ingreso de pacientes predominantemente portadores de patología respiratoria, por lo que los resultados pueden no ser susceptibles de generalizarse a otros entornos. Sin embargo, el presente trabajo contribuye al entendimiento del impacto de nuevas instalaciones de cuidados intensivos en medidas de resultados como los EA y complementa la información disponible del rendimiento de nuevos edificios para quienes participan en la transición de $\mathrm{UCl}$ con habitaciones compartidas a $\mathrm{UCl}$ con habitaciones individuales.

Este estudio sugiere que a pesar de contar con entornos con diseños "amigables" y soporte tecnológico de vanguardia, la seguridad del paciente crítico en habitaciones individuales debe ser tomada en cuenta. La transición a nuevas instalaciones, además de ir acompañada de cambios en los procesos de atención, requiere asignar el personal suficiente para satisfacer las nuevas demandas. La reubicación de una $\mathrm{UCl}$, proceso desgastante y demandante, puede resultar en riesgo para el paciente, sus familiares y el mismo personal de salud. Conseguir un estado estable en resultados de indicadores como los EA puede ser el punto de partida de una transición exitosa a una nueva área física.

\section{Declaración}

Los autores niegan conflictos de interés o financiamiento relacionados con la preparación de este trabajo. Su realización se apega a los requisitos éticos de la institución donde fue elaborado.

\section{Bibliografía}

1. Makary MA, Daniel M. Medical error-the third leading cause of death in the US. BMJ. 2016;353:12139.

2. Donchin Y, Gopher D, Olin M, Badihi Y, Biesky M, Sprung CL, et al. A look into the nature and causes of human errors in the intensivecare unit. Crit Care Med. 2003:12:143-147.

3. Donchin Y, Seagull FJ. The hostile environment of the intensivècare unit. Curr Opin Crit Care. 2002;8:316-320.

4. Zirming $\mathrm{C}$, Bosch $\mathrm{S}$. Building the evidence base for evidence-based design: editors' introduction. Environ Behav. 2008;40:147-150.

5. Ulrich RS, Zimring C, Zhu X, DuBose J, Seo HB, Choi YS, et al. A review of the research literature on evidence-based healthcare design. HERD. 2008:1:61-125.

6. Jongerden IP, Slooter AJ, Peelen LM, Wessels H, RamKesecioglu J, et al. Effect of intensive care environment on famit and patient satisfaction: a before-after study. Intensive Care Med. 2013 ; 39:1626-1634.

7. Lin FF, Foster M, Chaboyer W, Marshall A. Relocating an intensivereare unit: an exploratory qualitative study. Aust Crit Care. 2016;29:55-60.

8. Ferri M, Zygun DA, Harrison A, Stelfox HT. A study protocol for performance evaluation of a new academic intensive care unit facility: impact on patient care. BMJ Open. 2013;3:e003134.

9. Álvarez-Maldonado P, Cueto-Robledo G, Cerón-Díaz U, Pérez-Rosafes A, Navarro-Reynoso F, Cicero-Sabido R. Indicadores de calidad en una unidad de cuidados intensivos respiratorios. Análisis inicial de la base de datos DEDUCIR. Med Intensiva. 2012;36:518-520.

10. Álvarez-Maldonado $P$, Reding-Bernal A, Hernández-Solís A, Cicero-Sabido R. Impact of strategic planning, organizational culture imprint and care bundles to reduce adverse events in the ICU. Int J Qual Health Care. 2018.

11. Rothschild JM, Landrigan CP, Cronin JW, Kaushal R, Lockley $5 W$, Burdick E, et al. The Critical Care Safety Study: the incidence and hature of adverse events and serious medical errors in intensive care. Crit Care Med. 2005;33:1694-1700

12. Darley ESR, Vasant J, Leeming J, Hammond F, Matthews S, Albur M, et al. Impact of moving to a new hospital build, with a high proportion of single rooms, on healthcare-associated infections and outbreaks. JHosp Infect. 2018;98:191-193.

13. Parent JB. Moving technology into your new hospital: top 10 things to know. Advice for maintaining a sound strategy during a complex expansion. Healthc Inform. 2012;29:24-25.

14. Tussey KR, Yackzan SG, Davies CC. Moving into a new hospital tôwer: the MOVEE model. Nurse Manage. 2018;49:12-16.

15. Slosberg M, Nejati A, Evans J, Nanda U. Transitioning to a new facility: the crucial role of employee engagement. J Healthc Manag. 2018;63:63-77.

16. Maguire J, Burger K, O'Donnel P, Parnell L. Clinician perceptions of a changing hospital environment. HERD. 2013;6:69-79.

17. Munich RL. Transplanting an organization: how does culture matter.-Bull Menninger Clin. 2011;75:126-144.

18. Fairbrother $G$, Jones A, Rivas K. Changing model of nursing dare from individual patient allocation to team nursing in the acute inpatientienvironment. Contemp Nurse. 2010;35:202-220.

19. Neuraz A, Guérin $C$, Payet $C$, Polazzi S, Aubrun F, Dailler F, et al. Pâtient mortality is associated with staff resources and workload in the leU: a multicenter observational study. Crit Care Med. 2015;43:1587-1594

20. Verrillo SC, Winters BD. Review: continuous monitoring to detect failure to rescue in adult postoperative inpatients. Biomed Instrum Technol. 2018;52:281-287.

21. Eytan D, Goodwin A, Laussen P, Guerguerian AM. Insights from multi-dimensional physiological signals to predict and prevent cardiac arrests. Pediatr Crit Care Med. 2016;17:81-82 